\title{
High expression of CD47 in triple negative breast cancer is associated with epithelial-mesenchymal transition and poor prognosis
}

\author{
JINGPING YUAN ${ }^{1 *}$, XUEHUI SHI $^{2 *}$, CHUANG CHEN $^{3}$, HUIHUA HE $^{1}$, LIN LIU $^{1}$, JUAN WU $^{1}$ and HONGLIN YAN ${ }^{1}$ \\ ${ }^{1}$ Department of Pathology, Renmin Hospital of Wuhan University, Wuhan, Hubei 430060; \\ ${ }^{2}$ College of Chemistry and Molecular Sciences, Wuhan University, Wuhan, Hubei 430072; \\ ${ }^{3}$ Department of Breast and Thyroid Surgery, Renmin Hospital of Wuhan University, Wuhan, Hubei 430060, P.R. China
}

Received February 3, 2019; Accepted June 13, 2019

DOI: $10.3892 / 01.2019 .10618$

\begin{abstract}
CD47 functions as a dominant anti-engulfment signal on tumour cells and is overexpressed in various malignant tumours. However, the expression and functional significance of CD47 in triple-negative breast cancer (TNBC) is not completely understood. In the present study, CD47 was demonstrated to be overexpressed in TNBC solid tumours. Moreover, increased CD47 expression was significantly associated with an advanced tumour-node-metastasis stage, lymph node involvement and recurrence. Moreover, CD47 was an unfavourable and independent prognostic factor for 5-year disease-free survival in patients with TNBC. In addition, the expression of CD47 was associated with several markers of epithelial-mesenchymal transition (EMT). The present study was the first to demonstrate an association between increased expression of CD47 with EMT and poor prognosis of TNBC. Thus, CD47 may be a potential prognostic biomarker and therapeutic target for TNBC.
\end{abstract}

\section{Introduction}

At present, breast cancer is the most common type of cancer in women and one of the leading causes of cancer-associated mortality in women worldwide, despite improvements in diagnostic techniques and treatment modalities (1). The progression and metastasis of breast cancer are the main causes of mortality. Based on cellular markers reflecting the available targeted therapies, breast cancer is characterised as oestrogen

Correspondence to: Dr Honglin Yan, Department of Pathology, Renmin Hospital of Wuhan University, 99 Ziyang Road, Wuchang, Wuhan, Hubei 430060, P.R. China

E-mail: honglin1229@163.com

*Contributed equally

Key words: triple-negative breast cancer, CD47, prognosis, epithelial-mesenchymal transition receptor (ER)- or progesterone receptor (PR)-positive, human epidermal growth factor receptor 2 (HER2)-positive and triple-negative breast cancer (TNBC) (2). Targeted therapy can be implemented for ER/PR-positive and HER2-positive breast cancer; however, since TNBC is defined by the absence of the ER, PR and HER2 genes, there is no standard treatment for TNBC at present (3). The lack of targeted therapies and the poor prognosis in patients with TNBC has prompted major research efforts to identify new molecular targets of TNBC.

Studies have shown that functional changes in the immune system play an important role in the occurrence and development of carcinoma, where immunotherapy was a breakthrough point in cancer treatment (4). CD47, also known as integrin-associated protein, is a transmembrane protein that is highly expressed on the surface of various cancer cells. It interacts with thrombospondin-1, signal-regulatory protein- $\alpha$ (SIRP- $\alpha$ ) and other proteins to regulate various cellular functions, such as T cell activation and cell migration (5). Emerging evidence has indicated the function of CD47 as a dominant anti-engulfment signal on tumour cells, by binding with SIRP- $\alpha$ on phagocytic immune cells to prevent engulfment (6-8). Given that the expression of CD47 was inhibited in certain malignant tumours, the blockade of the CD47 anti-engulfment signal increased the phagocytosis of tumour cells in solid tumours and haematological malignancies (6). Therefore, CD47 is a protective therapeutic target on solid tumour cells.

Although breast cancer was not considered an immunogenic malignant tumour, certain studies have revealed an association between the intratumoural immune response and tumour progression (9-11). Since TNBC and HER2-positive breast cancer are highly proliferative, increased genomic instability and mutational burden may result in the exposure of a large number of tumour antigens and promote the anti-tumour immune response (12). Thus, TNBC and HER2-positive breast cancer were considered to be more immunogenic than other types of breast cancer (12). Although studies have shown the abnormal expression of CD47 in breast cancer cell lines, breast cancer stem cells, bone marrow, peripheral blood and the circulating tumour cells of breast cancer (13-17), most of these studies focused on the association between CD47 and breast cancer at the cytological level. There is little direct 
evidence of the expression of CD47 in breast cancer solid tumours, particularly in TNBC solid tumours, and its association with metastasis, survival rate and prognosis have rarely been reported.

Epithelial-mesenchymal transition (EMT) is a favourable explanation for the distant metastasis of breast cancer (18). The essential characteristics of EMT include the destruction of tight junctions and the loss of cell-to-cell contact, which leads to the loss of epithelial characteristics (such as the loss of the epithelial cell adhesion protein E-cadherin) and the acquisition of a mesenchymal morphology (such as the gain of the mesenchyme-associated molecule N-cadherin) (18). EMT can be induced by a variety of stimulants, such as cytokines and growth factor signalling molecules, and transforming growth factor- $\beta$ (TGF- $\beta$ ) is strongly involved in the induction of EMT in epithelial cells (including breast cancer epithelial cells) (19). However, the association between the expression of CD47 and EMT in breast cancer was rarely reported. In the present study, the expression of CD47 and its prognostic significance in TNBC solid tumours was evaluated, as well as the association between CD47 and EMT, in order to explore novel mechanisms for the development of TNBC therapies.

\section{Materials and methods}

Tissues. Formalin-fixed, paraffin-embedded tissue samples from 57 patients with TNBC, who underwent surgery for primary breast cancer, were randomly selected from the medical records of the Department of Pathology, Renmin Hospital of Wuhan University between August 2009-December 2010. The inclusion criteria were as follows: i) Received no treatment prior to surgery; and ii) female. Patient ages were in the range of 31-69 years (mean, 50.2 years; median, 42 years). Major clinicopathological parameters, including age, menopausal status, tumour-node-metastasis (TNM) stage, histological grade, lymph node metastasis and recurrence, are summarized in Table II. TNM stage was grouped according to the American Joint Committee on Cancer 7th Edition Cancer Staging Manual 2010 (20). The histological grade was classified in accordance with the 4th edition of the WHO histological grading (21). Each patient was followed up for 5 years after surgery and the survival times were recorded. A total of 40 cases of benign breast lesions were also randomly selected from Department of Pathology, Renmin Hospital of Wuhan University between August 2009 and December 2010 as controls. The ages were in the range of 19-64 years, with a mean age of 35 years. Written informed consent was obtained from the patients, and the study was approved by the Ethics Committee of Renmin Hospital of Wuhan University.

Immunohistochemistry (IHC). IHC was performed to detect CD47, E-cadherin, N-cadherin and TGF- $\beta$. Formalin-fixed, paraffin-embedded tissue samples were cut into $4 \mu \mathrm{m}$ sections and then deparaffinised with xylene twice for $10 \mathrm{~min}$ and rehydrated with $100 \%$ ethanol twice for $5 \mathrm{~min}, 95 \%$ ethanol twice for $2 \mathrm{~min}$ and $85 \%$ ethanol for $2 \mathrm{~min}$ at room temperature. Following that, the sections were blocked with $3 \% \mathrm{H}_{2} \mathrm{O}_{2}$ for $10 \mathrm{~min}$ at room temperature to block endogenous peroxidase activity and then subjected to antigen retrieval in citrate buffer (pH 6.0) at $98^{\circ} \mathrm{C}$ for $15 \mathrm{~min}$. This was followed by incubation with primary antibodies: Anti-CD47 (1:100; cat. no. sc-12730; Santa Cruz Biotechnology, Inc.); anti-E-cadherin (1:100; cat. no. ab1416; Abcam); anti-N-cadherin (1:500; cat. no. ab18203; Abcam); or anti-TGF- $\beta$ (1:100; sc-130348; Santa Cruz Biotechnology, Inc.), for $1 \mathrm{~h}$ at $37^{\circ} \mathrm{C}$. The slides were incubated with a biotinylated secondary antibody using the Dako LSAB2 system-horseradish peroxidase (cat. no. K0672; Agilent Technologies, Inc.) for $30 \mathrm{~min}$ at $37^{\circ} \mathrm{C}$. The reaction products were stained with 3,3'-diaminobenzidine at room temperature for 2-3 min and lightly counterstained with haematoxylin at room temperature for $2 \mathrm{~min}$. Sections incubated in PBS without a primary antibody were used as a blank control. The slides were examined under an Olympus light microscope (Olympus Corporation) at x200 and x400 magnification.

Staining evaluation. Immunohistochemical staining of all sections were evaluated by two independent pathologists (JY and $\mathrm{HH})$. The evaluating pathologists were blinded to the clinical data. In the present study, the expression of CD47 was mainly localised to the cytoplasm or cell membrane, which appeared yellow or brown. The degree of CD47 reactivity was scored by applying a semi-quantitative immunoreactivity scoring (IRS) system as described by Baccelli et al (13). The staining intensity was categorized into four grades: 0 , no immunostaining; 1 , weak staining; 2 , moderate staining; and 3 , strong staining. The percentage of positive cells was categorized into five grades: 0 , $0 \% ; 1,1-10 \% ; 2,11-50 \% ; 3,51-80 \%$; and $4,>80 \%$. The staining intensity and percentage of positive cells were multiplied to obtained an IRS, in the range of 0-12 for each individual case. A case was scored as positive for CD47 with an IRS between 7 and 12 and negative with an IRS between 0-6 (13).

E-cadherin-positive cells contained yellow or dark brown granules on the cell membrane. $\mathrm{N}$-cadherin-positive cells exhibited a yellow or dark brown colour in the cytoplasm or at the cellular membrane. According to the method described by Nakajima et al (22) and Liu et al (23), E-cadherin expression was considered to be normal if $\geq 90 \%$ of cancer cells exhibited a staining pattern similar to that in normal epithelial cells, and sections with $<90 \%$ of the cancer cells stained or with a complete absence of staining were classified as a reduced pattern. Therefore, $\geq 90 \%$ indicated positivity and $<90 \%$ indicated negativity for E-cadherin expression. Similarly, according to the method described by Liu et al (23), the proportion of positive cells $\geq 5 \%$ indicated positivity, whereas $<5 \%$ indicated negativity for $\mathrm{N}$-cadherin expression.

TGF- $\beta$ was primarily located in the cytoplasm or nucleus and was indicated by a yellow or brown colour. The immunohistochemical staining of tumour cells was evaluated semi-quantitatively, as described by Chen et al (24), based on: i) Staining intensity, where 0, No staining; 1, weak staining; 2 , moderate staining; and 3 , strong staining; and ii) extent of the staining, where $0, \leq 5 \% ; 1,6-25 \% ; 2,26-50 \% ; 3,51-75 \%$; and $4,76-100 \%$. The final score was obtained by multiplying the scores for both the staining intensity and the extent of the staining; a final score $\leq 2$ indicated negative staining, and $>2$ indicated positive staining.

Statistical analysis. The $\chi^{2}$ test was used to compare the CD47 expression between TNBC tissues and benign breast lesions, 
analyse the association between CD47 expression and the clinicopathological parameters, and evaluate the association between CD47 expression and EMT markers. Kaplan-Meier analysis and the log-rank test were used for survival analysis. The prognostic value of CD47 was estimated by univariate and multivariate Cox proportional hazard regression analysis. All statistical analyses were performed with SPSS software version 19.0 (IBM Corp.). $\mathrm{P}<0.05$ was considered to indicate a statistically significant difference. Data are presented as the mean \pm standard error of the mean. The experiments were repeated in triplicate.

\section{Results}

CD47 is highly expressed in TNBC tissues. The expression of CD47 was detected in 40 benign breast lesions and 57 TNBC tissues (Fig. 1A and B). In TNBC tissues, CD47 was mainly expressed in the cytoplasm or at the cell membrane in brown granules (Fig. 1A), and positive immunohistochemical expression of CD47 was found in 44/57 (77.2\%) cases. The immunoreactivity for CD47 in benign breast lesions was negative or weakly positive in the cytoplasm (Fig. 1B). The CD47-positive rate in 40 cases of benign breast lesions was $30 \%$ (12/40 cases). The CD47-positive rate was significantly higher in TNBC tissues than in benign breast lesions $\left(\chi^{2}=21.453 ; \mathrm{P}<0.001\right.$; Table I $)$.

Association between CD47 expression and clinicopathological parameters of TNBC. The association between CD47 expression and clinicopathological parameters were further analysed in 57 TNBC tissues (Table II). Positive staining for CD47 was significantly associated with advanced TNM stage $\left(\chi^{2}=7.241\right.$; $\mathrm{P}=0.027)$, lymph node involvement $\left(\chi^{2}=4.403 ; \mathrm{P}=0.036\right)$ and recurrence $\left(\chi^{2}=5.900 ; \mathrm{P}=0.015\right)$. The $\mathrm{CD} 47$-positive rate increased with increasing TNM stage (0, 71.1 and 94.4\%). The CD47-positive rate in patients with lymph node metastasis or recurrence was significantly higher than that in patients without lymph node metastasis or recurrence. There was no significant association between positive staining for CD47 and other clinicopathological parameters, such as patient age, menopause and histological grade $(\mathrm{P}>0.05)$.

Association between CD47 and EMT markers. Currently, EMT is a favourable explanation for the distant metastasis of epithelial cancers, including TNBC. The loss of E-cadherin and the gain of N-cadherin expression are markers of EMT. Consistent with previous reports, the expression of E-cadherin was decreased (Fig. 1C), whereas the expression of $\mathrm{N}$-cadherin was increased (Fig. 1D) in TNBC tissues. The TGF- $\beta$ pathway has been strongly implicated in inducing EMT in epithelial cells (17). Thus, the expression of TGF- $\beta$ was also examined in TNBC tissues (Fig. 1E).

The association between the expression of CD47 and EMT markers was investigated (Table III). The expression of CD47 was associated with decreased expression of E-cadherin $\left(\chi^{2}=4.414 ; \mathrm{P}=0.036\right)$ and increased expression of $\mathrm{N}$-cadherin $\left(\chi^{2}=9.216 ; \mathrm{P}=0.002\right) . \mathrm{CD} 47$ expression was associated with increased expression of TGF- $\beta\left(\chi^{2}=8.093 ; \mathrm{P}=0.004\right)$. These results suggested that the expression of CD47 may be involved in the process of EMT in TNBC.

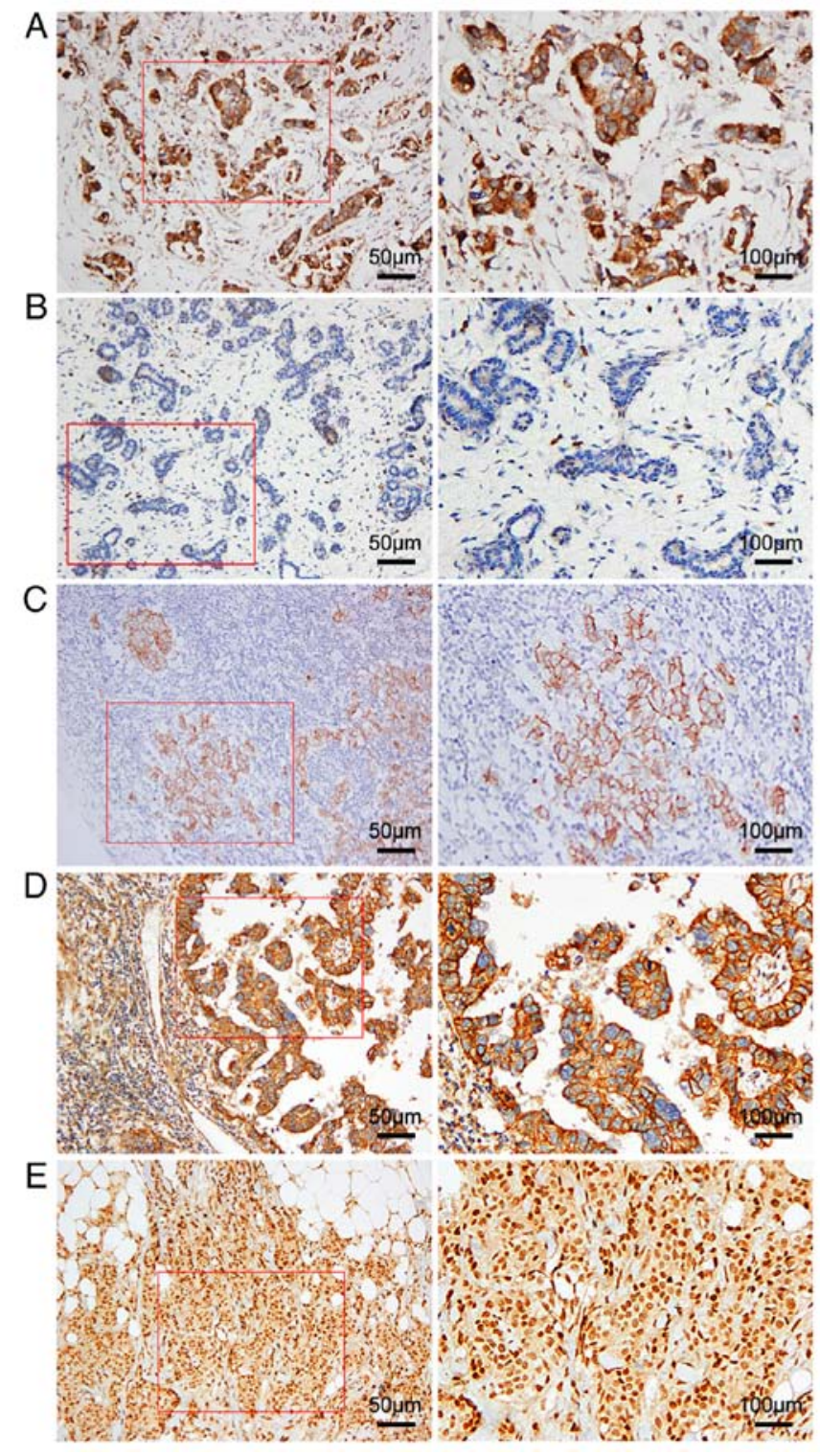

Figure 1. Immunohistochemical staining for CD47, E-cadherin, N-cadherin and TGF- $\beta$ in representative tissue specimens. The expression of CD47 in (A) TNBC tissues and in (B) benign breast lesions. The expression of (C) E-cadherin, (D) N-cadherin and (E) TGF- $\beta$ in TNBC tissues. 3,3'-Diaminobenzidine staining (brown) and nuclear counterstaining with haematoxylin (blue). Magnifications: left, x200; and right, $\mathrm{x} 400$. TNBC, triple-negative breast cancer; TGF- $\beta$, transforming growth factor- $\beta$.

Expression of CD47 is a marker of poor prognosis in TNBC. The prognostic value of CD47 was determined in 57 TNBC cases, following a 5-year follow-up. Kaplan-Meier analysis and the log-rank test showed a statistically significant association between a high expression level of CD47 and a low 5-year disease-free survival (5-DFS) time ( $\mathrm{P}=0.034$; Fig. 2), indicating that a high expression level of CD47 may serve as a novel marker of TNBC with a poor clinical outcome. To further validate the prognostic significance of this new parameter, CD47 and all clinicopathological features, including age, menopausal status, TNM stage, histological grade, lymph node metastasis and recurrence, were subjected to univariate and multivariate Cox proportional hazard regression analysis. As presented in Table IV, univariate Cox proportional hazard regression analysis confirmed that an advanced 
Table I. Expression of CD47 protein in breast benign lesions and triple-negative breast cancer tissues.

\begin{tabular}{lcccr}
\hline & & \multicolumn{3}{c}{ CD47 protein expression } \\
\cline { 3 - 5 } Groups & All cases, $\mathrm{n}$ & Positive, $\mathrm{n}(\%)$ & $\chi^{2}$ & P-value \\
\hline Breast benign lesions & 40 & $12(30.0)$ & 21.453 & $<0.001$ \\
Triple-negative breast cancer tissues & 57 & $44(77.2)$ & & \\
\hline
\end{tabular}

Table II. Association between CD47 protein expression and clinicopathological parameters of patients with triple-negative breast cancer.

\begin{tabular}{|c|c|c|c|c|c|}
\hline \multirow[b]{2}{*}{ Clinicopathological parameters } & \multirow[b]{2}{*}{ All cases, $\mathrm{n}$} & \multicolumn{4}{|c|}{ CD47 protein expression } \\
\hline & & Negative, $\mathrm{n}$ & Positive, n (\%) & $\chi^{2}$ & P-value \\
\hline Age, years & & & & 0.888 & 0.346 \\
\hline$<50$ & 33 & 9 & $24(72.7)$ & & \\
\hline$>50$ & 24 & 4 & $20(83.3)$ & & \\
\hline Menopause & & & & 0.150 & 0.698 \\
\hline After & 28 & 7 & $21(75.0)$ & & \\
\hline Before & 29 & 6 & $23(79.3)$ & & \\
\hline TNM & & & & 7.241 & 0.027 \\
\hline I & 1 & 1 & $0(0)$ & & \\
\hline II & 38 & 11 & $27(71.1)$ & & \\
\hline III & 18 & 1 & $17(94.4)$ & & \\
\hline Histological grade & & & & 0.331 & 0.848 \\
\hline G1 & 6 & 1 & $5(83.3)$ & & \\
\hline G2 & 27 & 7 & $20(74.1)$ & & \\
\hline G3 & 24 & 5 & $19(79.2)$ & & \\
\hline Lymph node metastasis & & & & 4.403 & 0.036 \\
\hline Yes & 32 & 4 & $28(87.5)$ & & \\
\hline No & 25 & 9 & $16(64.0)$ & & \\
\hline Recurrence & & & & 5.900 & 0.015 \\
\hline Yes & 30 & 3 & $27(83.3)$ & & \\
\hline No & 27 & 10 & $17(70.4)$ & & \\
\hline
\end{tabular}

TNM, Tumour-Node-Metastasis.

TNM stage $(\mathrm{P}<0.001 ; \mathrm{HR}=5.964 ; 95 \% \mathrm{CI}=2.709-13.129)$, the presence of lymph node metastasis $(\mathrm{P}<0.001 ; \mathrm{HR}=4.596$; 95\% CI=2.096-10.079), recurrence $(\mathrm{P}<0.001 ; \mathrm{HR}=64.448$; 95\% $\mathrm{CI}=9.512-436.670)$, and positive CD47 expression $(\mathrm{P}=0.042 ; \mathrm{HR}=2.464 ; 95 \% \mathrm{CI}=1.032-5.882)$ were significantly associated with a poor 5-DFS time in patients with TNBC. Multivariate statistical analysis confirmed that recurrence $(\mathrm{P}<0.001 ; \mathrm{HR}=91.009 ; 95 \% \mathrm{CI}=9.950-832.400)$ and positive CD47 expression $(\mathrm{P}=0.037 ; \mathrm{HR}=3.432 ; 95 \% \mathrm{CI}=1.079-10.922)$ were significant independent predictors of poor 5-DFS time.

\section{Discussion}

TNBC accounts for $15 \%$ of all breast cancer cases and is characterised by early relapse and metastasis (25). The ability of cancer cells to escape the innate and adaptive immune systems plays a key role in the formation of secondary (recurrent and/or metastatic) tumours $(26,27)$. Lehmann et al (28) reported that the loss of immune infiltration in tumours was associated with mortality in patients with TNBC. One of the main mechanisms regulating the escape of cancer cells from innate immunity is the expression of CD47, which interacts with SIRP- $\alpha$ on macrophages to prevent phagocytosis. Shuptrine et al (29) identified 709 genes that selectively regulated adaptive anti-tumour immunity in a syngeneic TNBC model, by developing an unbiased, in vivo, genome-wide RNA interference screening platform. Of the five genes with the greatest impact identified by the screening, CD47 had the greatest impact on the immune regulatory pathway (29).

CD47 was first described as a tumour antigen in human ovarian cancer in the 1980s (30). Since then, CD47 has been found to be highly expressed in various types of human cancer, 
Table III. Association between the expression of CD47 and other proteins.

\begin{tabular}{|c|c|c|c|c|}
\hline \multirow[b]{2}{*}{ Protein } & \multicolumn{2}{|c|}{$\begin{array}{l}\text { CD47 protein } \\
\text { expression }\end{array}$} & \multirow[b]{2}{*}{$\chi^{2}$} & \multirow[b]{2}{*}{ P-value } \\
\hline & Positive & Negative & & \\
\hline E-cadherin & & & 4.414 & 0.036 \\
\hline Positive, $\mathrm{n}$ & 13 & 8 & & \\
\hline Negative, $\mathrm{n}$ & 31 & 5 & & \\
\hline Positive rate, $\%$ & 29.55 & 61.54 & & \\
\hline $\mathrm{N}$-cadherin & & & 9.216 & 0.002 \\
\hline Positive, $\mathrm{n}$ & 38 & 6 & & \\
\hline Negative, $\mathrm{n}$ & 6 & 7 & & \\
\hline Positive rate, $\%$ & 86.36 & 46.15 & & \\
\hline TGF- $\beta$ & & & 8.093 & 0.004 \\
\hline Positive, $\mathrm{n}$ & 35 & 5 & & \\
\hline Negative, $\mathrm{n}$ & 9 & 8 & & \\
\hline Positive rate, $\%$ & 79.55 & 38.46 & & \\
\hline
\end{tabular}

${ }^{\mathrm{a}} \chi^{2}$ test. TGF- $\beta$, transforming growth factor- $\beta$.

including non-Hodgkin's lymphoma (31), acute myeloid leukaemia (32), hepatocellular carcinoma $(33,34)$ and bladder cancer (35), and its expression is closely associated with the differentiation, metastasis, survival and prognosis of tumours. Accordingly, CD47 is considered a biomarker of cancer, and its high expression is an indicator of poor clinical prognosis. In breast cancer, the abnormal expression of CD47 has been detected in various breast cancer-associated cells, including in breast cancer cell lines (36), breast cancer stem cells (17), bone marrow (14), peripheral blood (14) and circulating tumour cells $(13,16)$ of breast cancer. However, most studies have evaluated the association between CD47 and breast cancer at the cytological level, and few have reported on the expression of CD47 in solid breast cancer tumours, particularly TNBC. The present study was the first, to the best of our knowledge, to reveal the overexpression of CD47 in TNBC solid tumour tissues compared with its expression in benign breast lesions.

CD47 is a novel prognostic biomarker of certain malignant tumours. In circulating colorectal cancer cells, the upregulation of the CD47 gene was associated with distant metastasis as a potential immune escape mechanism (37). In non-small cell lung cancer, increased CD47 expression was associated with clinical staging, $\mathrm{T}$ classification, lymph node metastasis and distant metastasis (38). Consistent with the above reports, the overexpression of CD47 in TNBC was significantly associated with TNM stage, lymph node involvement and recurrence in the present study. In addition, high CD47 expression levels have been reported to be an unfavourable independent prognostic factor for 5-DFS time (14). A previous study on patients with breast cancer indicated the low survival rate of patients with high CD47 expression in the bone marrow and circulating tumour cells than that of patients with low CD47 expression (14). In the present study, Kaplan-Meier analysis and the log-rank test demonstrated a poor 5-DFS time in patients with TNBC and high expression levels of CD47. A

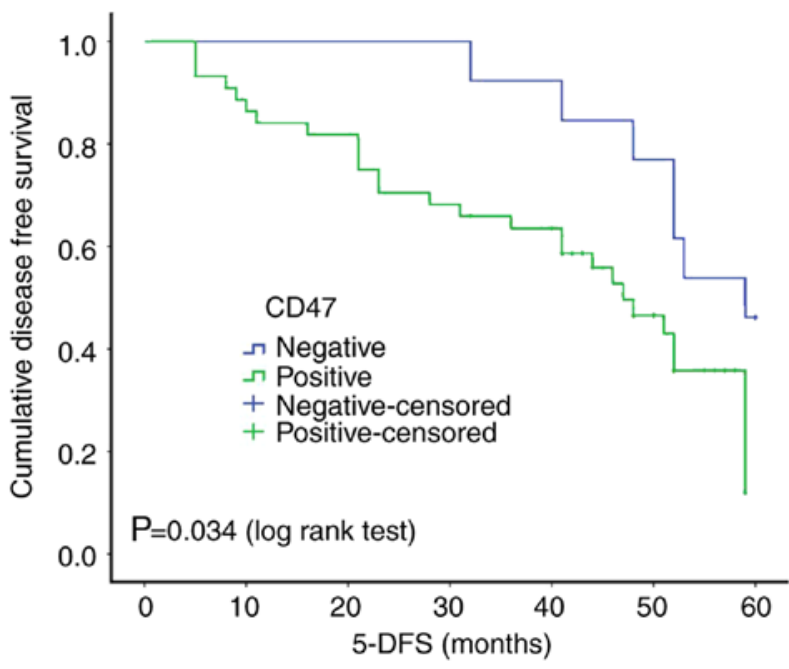

Figure 2. Effect of CD47 expression on the 5-DFS times of patients with TNBC. Kaplan-Meier survival analysis and log-rank test demonstrating the association between CD47 expression and the 5-DFS times of patients with TNBC. 5-DFS, 5-year disease-free survival; TNBC, triple-negative breast cancer.

study by Baccelli et al (13) demonstrated the co-expression of MET-CD47 as an independent prognostic factor for the overall survival (OS) of patients with breast cancer, and that this was associated with lymph node metastasis. It was found that the OS of patients with MET-CD47 double-positive luminal breast cancer was 10.3 years lower than in those with MET-CD47 double-negative expression. Further investigation in the present study, through multivariate statistical analysis, indicated the expression of CD47 in TNBC as an independent significant predictor of poor 5-DFS time. Overall, these findings revealed the potential of CD47 as a prognostic marker in TNBC.

High expression of CD47 in various tumours indicates poor prognosis; however, the underlying mechanism of the transcriptional regulation of CD47 remains unclear. EMT is regulated by a series of EMT-induced transcription factors that promote immune escape and drug resistance (39). Noman et al (40) reported the upregulation of CD47 in various EMT-activated human breast cancer cells and in the inhibition of phagocytosis. Li et al (41) demonstrated the induction of EMT by CD47, through the modulation of E-cadherin and N-cadherin, which contribute to the invasion of high-grade serous ovarian carcinoma. This suggests a potential association between CD47 expression and EMT. Consequently, the association between CD47 expression in TNBC and several markers of EMT was evaluated in the present study. The expression of CD47 was associated with high expression of $\mathrm{N}$-cadherin and TGF- $\beta$, in contrast with decreased expression of E-cadherin. Thus, the expression of CD47 may be involved in the process of EMT in TNBC. Mechanistically, SNAI1 and ZEB1 have been reported to be major regulators of CD47 (40). The overexpression of SNAI1 or ZEB1 in human breast cancer epithelial cells results in the activation of EMT and the upregulation of CD47, by binding directly to the E-box-2 and E-box-3 motifs of the human CD47 proximal promoter (40). This indicates an EMT-dependent upregulation of CD47 in breast cancer. On the other hand, Shinohara et al (42) demonstrated the co-localization of CD47 with E-cadherin at cell-cell adhesion sites in mouse 
Table IV. Univariate and multivariate Cox proportional hazard regression analysis of 5-year disease-free survival times of patients with triple-negative breast cancer.

\begin{tabular}{|c|c|c|c|c|c|c|c|c|}
\hline \multirow[b]{3}{*}{ Variable } & \multicolumn{4}{|c|}{ Univariate } & \multicolumn{4}{|c|}{ Multivariate } \\
\hline & \multirow[b]{2}{*}{ HR } & \multicolumn{2}{|c|}{$95 \% \mathrm{CI}$} & \multirow[b]{2}{*}{ P-value } & \multirow[b]{2}{*}{ HR } & \multicolumn{2}{|c|}{$95 \% \mathrm{CI}$} & \multirow[b]{2}{*}{ P-value } \\
\hline & & Lower & Upper & & & Lower & Upper & \\
\hline Age $(\leq 50$ vs. $>50)$ & 1.113 & 0.561 & 2.207 & 0.760 & 0.372 & 0.098 & 1.414 & 0.147 \\
\hline Menopause (after vs. before) & 0.698 & 0.355 & 1.373 & 0.298 & 0.386 & 0.111 & 1.340 & 0.134 \\
\hline TNM stage (I and II vs. III) & 5.964 & 2.709 & 13.129 & $<0.001$ & 1.361 & 0.524 & 3.531 & 0.527 \\
\hline Histological grade (grade 1 and 2 vs. grade 3 ) & 1.551 & 0.790 & 3.048 & 0.203 & 1.594 & 0.760 & 3.340 & 0.217 \\
\hline Lymph node metastasis (no vs. yes) & 4.596 & 2.096 & 10.079 & $<0.001$ & 2.255 & 0.814 & 6.243 & 0.118 \\
\hline Recurrence (no vs. yes) & 64.448 & 9.512 & 436.670 & $<0.001$ & 91.009 & 9.950 & 832.400 & $<0.001$ \\
\hline CD47 (negative vs. positive) & 2.464 & 1.032 & 5.882 & 0.042 & 3.432 & 1.079 & 10.922 & 0.037 \\
\hline
\end{tabular}

CI, confidence interval; HR, hazard ratio; TNM, Tumour-Node-Metastasis.

breast cancer-derived epithelial cells. Since E-cadherin was known to be involved in the actin cytoskeleton of epithelial cells, CD47 was found to reorganize the actin cytoskeleton to participate in the regulation of cell-cell adhesion and cell migration (42). Based on the findings of the present study and those from the literature, the involvement of CD47 in the process of EMT in TNBC is speculated. Whether CD47 promotes EMT in epithelial cells, or whether EMT-associated transcription factors or proteins regulate the expression of CD47, is yet to be determined.

At present, there are few studies on the expression of CD47 in TNBC solid tumours and its association with EMT. In the present study, the overexpression of CD47 was demonstrated in TNBC, which was associated with advanced TNM stage, lymph node involvement, recurrence and a reduced 5-DFS time. In addition, the expression of CD47 was associated with several EMT markers in TNBC. Overall, these findings suggest the potential of CD47 as a prognostic marker and therapeutic target for TNBC.

However, some limitations of the present study should be acknowledged. Firstly, the sample size is small. The sample number will be increased in order to explore the role and mechanism of CD47 in the progression of breast cancer in the future. Secondly, the association between CD47 expression and prognosis was evaluated using 5-DFS data, rather than OS. Since DFS is not directly associated with OS, the effect of CD47 expression on OS remains a topic for future studies. Therefore, the results of the study should be interpreted with caution, and further validation is warranted with a larger sample size and longer clinical follow-up time.

\section{Acknowledgements}

Not applicable.

\section{Funding}

This work was supported by grants from: The National Natural Science Foundation of China (grant no. 31600866); the Science and Technology Planning Project of Wuhan (grant no. 2017060201010172); and the Guidance Foundation of Renmin Hospital of Wuhan University (grant no. RMYD2018M27).

\section{Availability of data and materials}

The datasets used and/or analysed during the present study are available from the corresponding author on reasonable request.

\section{Authors' contributions}

CC, LL and JW collected the samples and performed the statistical analysis; JY and HH evaluated the immunohistochemical staining of all sections; JY, XS and HY designed the study and wrote the manuscript; HY revised the manuscript. All authors read and approved the final manuscript.

\section{Ethics approval and consent to participate}

The present study was approved by the Ethics Committee of Renmin Hospital of Wuhan University (Wuhan, China) (approval no. WDRY2019-K010). Patients provided written informed consent for the use of their tissues in the present study.

\section{Patient consent for publication}

Not applicable.

\section{Competing interests}

The authors declare that they have no competing interests.

\section{References}

1. Hortobagyi GN, de la Garza Salazar J, Pritchard K, Amadori D, Haidinger R, Hudis CA, Khaled H, Liu MC, Martin M, Namer M, et al: The global breast cancer burden: Variations in epidemiology and survival. Clin Breast Cancer 6: 391-401, 2005.

2. Kumar P and Aggarwal R: An overview of triple-negative breast cancer. Arch Gynecol Obstet 293: 247-269, 2016.

3. Engebraaten O, Vollan HKM and Borresen-Dale AL: Triple-negative breast cancer and the need for new therapeutic targets. Am J Pathol 183: 1064-1074, 2013. 
4. Couzin-Frankel J: Breakthrough of the year 2013. Cancer immunotherapy. Science 342: 1432-1433, 2013.

5. Liu XJ, Kwon H, Li ZH and Fu YX: Is CD47 an innate immune checkpoint for tumor evasion? J Hematol Oncol 10: 12, 2017.

6. McCracken MN, Cha AC and Weissman IL: Molecular pathways: Activating T cells after cancer cell phagocytosis from blockade of CD47 'don't eat me' signals. Clin Cancer Res 21: 3597-3601, 2015.

7. Willingham SB, Volkmer JP, Gentles AJ, Sahoo D, Dalerba P, Mitra SS, Wang J, Contreras-Trujillo H, Martin R, Cohen JD, et al The CD47-signal regulatory protein alpha (SIRPa) interaction is a therapeutic target for human solid tumors. Proc Natl Acad Sci USA 109: 6662-6667, 2012.

8. Vonderheide RH: CD47 blockade as another immune checkpoint therapy for cancer. Nat Med 21: 1122-1123, 2015.

9. Gingras I, Azim HA Jr, Ignatiadis M and Sotiriou C: Immunology and breast cancer: Toward a new way of understanding breast cancer and developing novel therapeutic strategies. Clin Adv Hematol Oncol 13: 372-382, 2015.

10. Vonderheide RH, Domchek SM and Clark AS: Immunotherapy for breast cancer: What are we missing? Clin Cancer Res 23 2640-2646, 2017.

11. Santa-Maria CA, Park SJ, Jain S and Gradishar WJ: Breast cancer and immunology: Biomarker and therapeutic developments. Expert Rev Anticancer Ther 15: 1215-1222, 2015.

12. Bianchini G, Balko JM, Mayer IA, Sanders ME and Gianni L: Triple-negative breast cancer: Challenges and opportunities of a heterogeneous disease. Nat Rev Clin Oncol 13: 674-690, 2016.

13. Baccelli I, Stenzinger A, Vogel V, Pfitzner BM, Klein C, Wallwiener M, Scharpff M, Saini M, Holland-Letz T, Sinn HP, et al: Co-expression of MET and CD47 is a novel prognosticator for survival of luminal breast cancer patients. Oncotarget 5: 8147-8160, 2014.

14. Nagahara M, Mimori K, Kataoka A, Ishii H, Tanaka F, Nakagawa T, Sato T, Ono S, Sugihara K and Mori M: Correlated expression of CD47 and SIRPA in bone marrow and in peripheral blood predicts recurrence in breast cancer patients. Clin Cancer Res 16: 4625-4635, 2010.

15. Bener G, J Félix A, Sánchez de Diego C, Pascual Fabregat I, Ciudad CJ and Noé V: Silencing of CD47 and SIRP $\alpha$ by Polypurine reverse hoogsteen hairpins to promote MCF-7 breast cancer cells death by PMA-differentiated THP-1 cells. BMC Immunol 17: 32, 2016 .

16. Baccelli I, Schneeweiss A, Riethdorf S, Stenzinger A, Schillert A, Vogel V, Klein C, Saini M, Bauerle T, Wallwiener M, et al: Identification of a population of blood circulating tumor cells from breast cancer patients that initiates metastasis in a xenograft assay. Nat Biotechnol 31: 539-544, 2013.

17. Kaur S, Elkahloun AG, Singh SP, Chen OR, Meerzaman DM, Song T, Manu N, Wu W, Mannan P, Garfield SH and Roberts DD: A function-blocking CD47 antibody suppresses stem cell and EGF signaling in triple-negative breast cancer. Oncotarget 7: 10133-10152, 2016.

18. Wu Y, Sarkissyan M and Vadgama JV: Epithelial-mesenchymal transition and breast cancer. J Clin Med 5: E13, 2016.

19. Massagué J: TGF $\beta$ signalling in context. Nat Rev Mol Cell Bio 13: 616-630, 2012.

20. Edge SB, Byrd DR, Compton CC, Fritz AG, Greene FL and Trotti A: AJCC Cancer Staging Manual. 7th edition. Springer, New York, NY, 2010.

21. Lakhani SR, Ellis IO, Schnitt SJ, Tan PH and Vijver MJ: WHO Classification of Tumours of the Breast. In: WHO Classification of Tumours. 4th Edition, IARC Press, Lyon, France, 2012.

22. Nakajima S, Doi R, Toyoda E, Tsuji S, Wada M, Koizumi M, Tulachan SS, Ito D, Kami K, Mori T, et al: N-cadherin expression and epithelial-mesenchymal transition in pancreatic carcinoma. Clin Cancer Res 10: 4125-4133, 2004

23. Liu GL, Yang HJ, Liu T and Lin YZ: Expression and significance of E-cadherin, $\mathrm{N}$-cadherin, transforming growth factor- $\beta 1$ and twist in prostate cancer. Asian Pac J Trop Med 7: 76-82, 2014.

24. Chen K, Wei H, Ling S and Yi C: Expression and significance of transforming growth factor- $\beta 1$ in epithelial ovarian cancer and its extracellular matrix. Oncol Lett 8: 2171-2174, 2014.

25. Newman LA, Reis-Filho JS, Morrow M, Carey LA and King TA: The 2014 society of surgical oncology Susan G. Komen for the cure symposium: Triple-negative breast cancer. Ann Surg Oncol 22: 874-882, 2015
26. Pardoll DM: The blockade of immune checkpoints in cancer immunotherapy. Nat Rev Cancer 12: 252-264, 2012.

27. Gajewski TF, Schreiber $\mathrm{H}$ and $\mathrm{Fu} \mathrm{YX}$ : Innate and adaptive immune cells in the tumor microenvironment. Nat Immunol 14 1014-1022, 2013.

28. Lehmann BD, Bauer JA, Chen X, Sanders ME, Chakravarthy AB, Shyr Y and Pietenpol JA: Identification of human triple-negative breast cancer subtypes and preclinical models for selection of targeted therapies. J Clin Invest 121: 2750-2767, 2011.

29. Shuptrine CW, Ajina R, Fertig EJ, Jablonski SA, Kim Lyerly H, Hartman ZC and Weiner LM: An unbiased in vivo functional genomics screening approach in mice identifies novel tumor cell-based regulators of immune rejection. Cancer Immunol Immunother 66: 1529-1544, 2017.

30. Knauf S, Kalwas J, Helmkamp BF, Harwell LW, Beecham J and Lord EM: Monoclonal antibodies against human ovarian tumor associated antigen NB/70K: Preparation and use in a radioimmunoassay for measuring NB/70K in serum. Cancer Immunol Immunother 21: 217-225, 1986.

31. Chao MP, Alizadeh AA, Tang C, Myklebust JH, Varghese B, Gill S, Jan M, Cha AC, Chan CK, Tan BT, et al: Anti-CD47 antibody synergizes with rituximab to promote phagocytosis and eradicate non-Hodgkin lymphoma. Cell 142: 699-713, 2010.

32. Galli S, Zlobec I, Schurch C, Perren A, Ochsenbein AF and Banz Y: CD47 protein expression in acute myeloid leukemia: A tissue microarray-based analysis. Leuk Res 39: 749-756, 2015.

33. Lee TK, Cheung VC, Lu P, Lau EY, Ma S, Tang KH, Tong M, Lo $\mathrm{J}$ and $\mathrm{Ng}$ IO: Blockade of CD47-mediated cathepsin S/protease-activated receptor 2 signaling provides a therapeutic target for hepatocellular carcinoma. Hepatology 60: 179-191, 2014

34. Xiao Z, Chung H, Banan B, Manning PT, Ott KC, Lin S, Capoccia BJ, Subramanian V, Hiebsch RR, Upadhya GA, et al: Antibody mediated therapy targeting CD47 inhibits tumor progression of hepatocellular carcinoma. Cancer Lett 360: 302-309, 2015.

35. Chan KS, Espinosa I, Chao M, Wong D, Ailles L, Diehn M, Gill H, Presti J Jr, Chang HY, van de Rijn M, et al: Identification, molecular characterization, clinical prognosis, and therapeutic targeting of human bladder tumor-initiating cells. P Natl Acad Sci USA 106: 14016-14021, 2009.

36. Manna PP and Frazier WA: CD47 mediates killing of breast tumor cells via $\mathrm{Gi}$-dependent inhibition of protein kinase $\mathrm{A}$. Cancer Res 64: 1026-1036, 2004.

37. Steinert G, Schölch S, Niemietz T, Iwata N, García SA, Behrens B, Voigt A, Kloor M, Benner A, Bork U, et al: Immune escape and survival mechanisms in circulating tumor cells of colorectal cancer. Cancer Res 74: 1694-1704, 2014.

38. Zhao H, Wang J, Kong X, Li E, Liu Y, Du X, Kang Z, Tang Y, Kuang Y, Yang Z, et al: CD47 promotes tumor invasion and metastasis in non-small cell lung cancer. Sci Rep 6: 29719, 2016.

39. Nieto MA, Huang RY, Jackson RA and Thiery JP: Emt: 2016. Cell 166: 21-45, 2016.

40. Noman MZ, Van Moer K, Marani V, Gemmill RM, Tranchevent LC, Azuaje F, Muller A, Chouaib S, Thiery JP, Berchem G and Janji B: CD47 is a direct target of SNAI1 and ZEB1 and its blockade activates the phagocytosis of breast cancer cells undergoing EMT. Oncoimmunology 7: e1345415, 2018.

41. Li Y, Lu S, Xu Y, Qiu C, Jin C, Wang Y, Liu Z and Kong B: Overexpression of $\mathrm{CD} 47$ predicts poor prognosis and promotes cancer cell invasion in high-grade serous ovarian carcinoma. Am J Transl Res 9: 2901-2910, 2017.

42. Shinohara M, Ohyama N, Murata Y, Okazawa H, Ohnishi H, Ishikawa O and Matozaki T: CD47 regulation of epithelial cell spreading and migration, and its signal transduction. Cancer Sci 97: 889-895, 2006.

This work is licensed under a Creative Commons Attribution-NonCommercial-NoDerivatives 4.0 International (CC BY-NC-ND 4.0) License. 